\title{
Thermal Collapse of Spin Polarization in Half-Metallic Ferromagnets
}

\author{
M. Ležaić, ${ }^{*}$ Ph. Mavropoulos, ${ }^{\dagger}$ J. Enkovaara, G. Bihlmayer, and S. Blügel \\ Institut für Festkörperforschung, Forschungszentrum Jülich, D-52425 Jülich, Germany
}

(Received 13 December 2005; published 13 July 2006)

\begin{abstract}
We propose two novel approaches to study the temperature dependence of the magnetization and the spin polarization at the Fermi level in magnetic compounds, and apply them to half-metallic ferromagnets. We reveal a new mechanism, where the hybridization of states forming the half-metallic gap depends on thermal spin fluctuations and the polarization can drop abruptly at temperatures much lower than the Curie point. We verify this for NiMnSb by ab initio calculations. The thermal properties are studied by mapping $a b$ initio results to an extended Heisenberg model which includes longitudinal fluctuations and is solved by a Monte Carlo method.
\end{abstract}

DOI: 10.1103/PhysRevLett.97.026404

Half-metallic ferromagnets (HMFs) are ferromagnetic metallic compounds showing, in the ideal case and at zero temperature, a spin polarization of $P=100 \%$ at the Fermi level $E_{F}$. This means that the spin-resolved density of states (DOS) shows a metallic character only for one spin direction (usually majority spin), with energy bands crossing $E_{F}$; contrary to this, the other spin direction (minority spin) behaves like an insulator with $E_{F}$ inside a band gap. This exotic behavior has inspired extensive research, since the extreme spin polarization suggests that HMFs are ideal for inducing and manipulating the transport of spinpolarized electrons.

Since the original introduction of HMFs in 1983 [1], their properties have been explored in depth. Theoretical studies have focused mainly on their ground-state properties: the magnetic moments and the origin of the gap [2]. The stability of the gap was studied with respect to spinorbit coupling [3], surface and interface states [4], tetragonal distortion [5], the presence of defects [6], and the appearance of nonquasiparticle states [7]. The extreme spin polarization has also been verified experimentally in a few compounds [8]. However, it is clear that the ideal half-metallic property $(P=100 \%)$ cannot be present at elevated temperatures $T$. Fluctuations of magnetic moments mix the two spin channels, and at latest at the Curie point, $T_{C}$, the polarization vanishes together with the magnetization. Thus, for applications one seeks HMFs with $T_{C}$ significantly higher than room temperature, reasonably assuming that the $T$ dependence of the spin polarization, $P(T)$, approximately follows the magnetization, i.e., $P(T) \propto M(T)$ [9]. But the theoretical study of $P(T)$ is far from trivial. Material-specific, first-principles calculations based on density-functional theory (DFT) capture the physics of hybridizations and bonding essential to the half-metallic property [2], but are designed in principle for the ground state and not for excited state properties. Nevertheless, adiabatic spin dynamics can be approximated within DFT, with successful applications in the prediction of $T_{C}$ [10-14].
PACS numbers: 71.20.Be, 71.20.Lp, 75.30.Et, 75.50.Cc

In many cases, HMFs have more than one magnetic atom per unit cell. For example, NiMnSb, a half-Heusler compound and prototypical example of all HMFs, has two magnetic atoms: $\mathrm{Mn}$ and Ni. It is the $d$ - $d$ hybridization between the Mn and Ni minority states that opens the halfmetallic gap [2], at least at $T=0$. At higher temperatures, directional fluctuations of local moments reduce the magnetization. In one-component systems it is well known that the magnetic configuration at each instant shows some degree of short-range order: small regions present almost collinear magnetic moments, with a local spin quantization axis $\hat{e}_{\text {loc }}$ not necessarily parallel to the average moment (the global axis $\hat{e}_{\text {glob }}$ ), while the low-energy, longwavelength fluctuations are more significant for the decrease of the magnetization. This behavior continues up to and even above $T_{C}$. However, rather little is known for multicomponent systems, when the sublattices are coupled with different strength so that they can lose the magnetic order at different temperatures.

We follow two approaches to the excited states, based on DFT (one in connection to an extended Heisenberg model, and one within mean-field theory), in order to elucidate the problem of magnetization and polarization at $T>0$. Our focus is on NiMnSb, but the method is applicable to any multicomponent system. We conclude that the hypothesis $P(T) \propto M(T)$ is not valid. Instead, $P(T)$ can fall off much faster than $M(T)$. Furthermore, for NiMnSb we find that the Ni moment disorders already at very low $T$, showing a susceptibility peak at $50 \mathrm{~K}$.

We expect that the decrease of $P$ in the presence of noncollinear fluctuations arises due to three mechanisms: (1) A globally noncollinear effect: The local axis $\hat{e}_{\mathrm{loc}}$ of a region with short-range order is in general not parallel $\hat{e}_{\text {glob }}$. Thus, there is always a projection of locally spinup states to the globally spin-down direction in the gap region. (2) A locally noncollinear effect: The short-range order is not perfect, since the spin axis of each atom varies with respect to that of its neighbors. This effect can be more significant in multicomponent systems. Its result is 
that, even in a local spin frame of reference, spin-up wave functions of each atom are partly projected into the spindown states of its neighbors within the gap, so that $P$ is diminished. In the presence of only the first effect, $P(T)$ is expected to follow the average magnetization $M(T)$; the second effect arises in addition to the first, and $P(T)$ is even further reduced. (3) A hybridization effect (change in hybridization strength), which has been overlooked so-far, leads to a closure of the gap by shifting the conduction and valence band edges. In Fig. 1(a) we show schematically how the three mechanisms affect the minority-spin DOS. Naturally, changes in the majority DOS also occur and charge neutrality is conserved.

We turn now to the specific system NiMnSb. We find by first-principles calculations that, in the ground state, the Mn local magnetic moment is large $\left(M_{\mathrm{Mn}}=3.7 \mu_{B}\right)$, while the Ni local moment is much smaller $\left(M_{\mathrm{Ni}}=0.26 \mu_{B}\right)$ and originates mainly from the hybridization of the $d$ orbitals of $\mathrm{Mn}$ with the ones of $\mathrm{Ni}$ (the same mechanism which opens the half-metallic gap [2]). In particular, the $d$-d hybridization causes some transfer of weight from $\mathrm{Mn}$ to $\mathrm{Ni}$ in the unoccupied states and vice versa in the occupied ones. Thus, the Ni moment is formed, while the Mn moment is reduced. Consequently, the Ni moment does not really arise from strong intra-atomic exchange interactions (Hund's rule), and its formation lies at a much lower energy scale than that of the Mn moment. We verify this conclusion by performing constrained DFT [15] calculations: when the Mn magnetic moment is forced by a magnetic field to form an angle to its Mn neighbors, it changes very little, even in an artificial antiferromagnetic configuration. Contrary to this, the Ni moment vanishes already when it is constrained at an angle of $90^{\circ}$ with respect to the Mn moment, or when the Mn moments are placed in an artificial antiferromagnetic order.
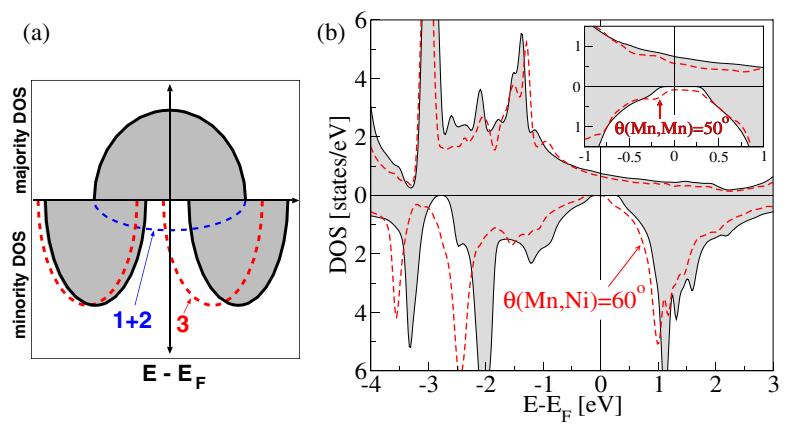

FIG. 1 (color online). (a) Modification of spin-down DOS around $E_{F}$ reducing the spin polarization due to mechanisms (1)(3) as discussed in text. (b) DOS of NiMnSb in the ground state (solid line) and in the state when the $\mathrm{Ni}$ moment has been constrained to an angle of $60^{\circ}$ with respect to the Mn moment (dashed line). (Inset) Dashed line: Here, Mn moments of neighboring layers along [001] direction form an angle of $50^{\circ}$ with respect to each other, while the Ni moments are allowed to relax. DOS are projected to the global frame.
We approach the finite-temperature properties using the adiabatic approximation for the calculation of magnon spectra [16]. We employ the full-potential linearized augmented plane-wave method as implemented in the FLEUR code [17] within the generalized gradient approximation [18] to DFT. Total-energy calculations of frozen magnons, performed at the equilibrium lattice constant of $5.9 \AA$, on a dense mesh of 2745 wave vectors $\vec{q}$ and $4096 \vec{k}$ points, provide the magnon dispersion relations $E(\vec{q})$. A subsequent Fourier transform yields the exchange constants $J_{i j}$ between sites $i$ and $j(\in\{\mathrm{Ni}, \mathrm{Mn}\})$ [10], mapping the system to a classical Heisenberg model. Thermodynamic quantities such as the magnetization $M(T)$, susceptibility $\chi(T)$, and Curie point $T_{C}$ can be found within this model by a Monte Carlo method [19]. In multicomponent systems we also consider the sublattice susceptibility $\chi_{n}$ and magnetization $M_{n}$ ( $n$ is a sublattice index). A peak of $\chi_{n}(T)$ signals the release of the corresponding degrees of freedom; i.e., the sublattice magnetization is randomized to a great extent. The total susceptibility $\chi(T)$ presents a peak at $T_{C}$.

While the Mn moment can be treated within the Heisenberg model as having a fixed absolute value and fluctuating only in its direction, for the weak Ni moment the longitudinal fluctuations are energetically as relevant as the transversal ones. These considerations require an extension of the traditional Heisenberg model allowing fluctuations of the magnitude of $M_{\mathrm{Ni}}$. This is possible, since the energy needed for constraining the Ni moment can be calculated within DFT and fitted well by a fourth-order function. Within this approximation, at each $\mathrm{Ni}$ site $i$ the neighboring atoms, placed at sites $j$, act as an exchange field $\vec{B}_{i}=\sum_{j} J_{i j} \vec{M}_{j}$ polarizing the $\mathrm{Ni}$ atom. Thus, the energy expression for the $\mathrm{Ni}$ atom at $i$ includes the magnitude of the local moment $M_{i}$ and the neighbor-induced polarizing field, and the total Hamiltonian reads

$$
H=-\frac{1}{2} \sum_{i, j} J_{i j} \vec{M}_{i} \cdot \vec{M}_{j}+\sum_{i \in\{\mathrm{Ni}\}}\left(a M_{i}^{2}+b M_{i}^{4}-\vec{B}_{i} \cdot \vec{M}_{i}\right) .
$$

Both the constants $a=18.4 \mathrm{mRy} / \mu_{B}^{2}$ and $b=$ $42.6 \mathrm{mRy} / \mu_{B}^{4}$, which are fitted to the $a b$ initio total-energy results, are positive, giving an energy minimum at $M_{i}=0$ if the neighbors have a zero net contribution, i.e., if $\vec{B}_{i}=0$. The second sum of Eq. (1) is applied to all Ni sites $i$ in the Monte Carlo calculation, on top of the first part, which is the usual Heisenberg expression. Note that, although the $\mathrm{Ni}$ moment is small and resulting from hybridization, a remnant of intra-atomic exchange still exists in $\mathrm{Ni}$, giving an enhanced on-site susceptibility. This assists the local moment formation and is reflected in the values of $a$ and $b$. The system can be regarded as an alloy of a strongly magnetic subsystem (Mn) with a paramagnetic subsystem with Stoner-enhanced susceptibility (Ni) [20].

After calculating the exchange parameters $J_{i j}$ (our results agree with those of Ref. [12]), the Monte Carlo 
calculation according to Eq. (1) yields the sublattice magnetizations $M_{\mathrm{Mn}}(T)$ and $M_{\mathrm{Ni}}(T)$ and the susceptibilities $\chi_{\mathrm{Mn}}(T)$ and $\chi_{\mathrm{Ni}}(T)$, shown in Fig. 2(a). Evidently, the overall thermodynamics are governed by the mighty $\mathrm{Mn}$ moment. The phase transition is clearly seen by the peak in the susceptibility $\chi(T)$, which grossly coincides with the Mn sublattice susceptibility $\chi_{\mathrm{Mn}}(T)$. A value of $T_{C} \approx$ $860 \mathrm{~K}$ is deduced, verified also by the method of cumulant expansion [19], and lies between the value of $940 \mathrm{~K}$ calculated in [14] and the experimental value of $T_{C}=730 \mathrm{~K}$. The surprising feature, however, is the behavior of $M_{\mathrm{Ni}}(T)$ and $\chi_{\mathrm{Ni}}(T)$. Already at low temperatures, around $50 \mathrm{~K}$, $M_{\mathrm{Ni}}(T)$ shows a rapid drop and $\chi_{\mathrm{Ni}}(T)$ a corresponding narrow peak. If we exclude the longitudinal fluctuations and work within the traditional Heisenberg model, an unpronounced behavior can be seen [broad maximum in $\chi_{\mathrm{Ni}}^{\text {transv }}(T)$ at around $T=300 \mathrm{~K}$ in Fig. 2(a)]. These results show that the Ni sublattice magnetic order is lost to a great extent. This behavior is traced back to the comparatively weak exchange constants $J_{i j}$ of the Ni moments to the neighboring atoms, as we found by the ab initio calculations.

Only a few experimental data exist on the $T$ dependence of $M_{\mathrm{Ni}}$. Neutron scattering [21] at 15 and $260 \mathrm{~K}$ shows a steady $M_{\mathrm{Ni}}\left(0.18 \mu_{B}\right)$, but a decreasing $M_{\mathrm{Mn}}$ (from $3.79 \mu_{B}$ to $\left.3.55 \mu_{B}\right)$. We do not know of any theoretical reason for the magnetically weak Ni to keep its moment while the strong $M_{\mathrm{Mn}}$ drops. X-ray magnetic circular dichroism measurements [22] show a fast drop of both $M_{\mathrm{Mn}}$ and $M_{\mathrm{Ni}}$ at $80 \mathrm{~K}$ to half their ground-state value, and a leveloff thereon. Such behavior is most unusual, but as stated in [22], surface effects possibly complicate the interpretation of dichroism data.

It is well known that the classical Heisenberg model cannot capture the very low-energy spectrum of the quantum Heisenberg model; therefore the low- $T$ behavior of $M$ is usually not well reproduced. However, since the longi-
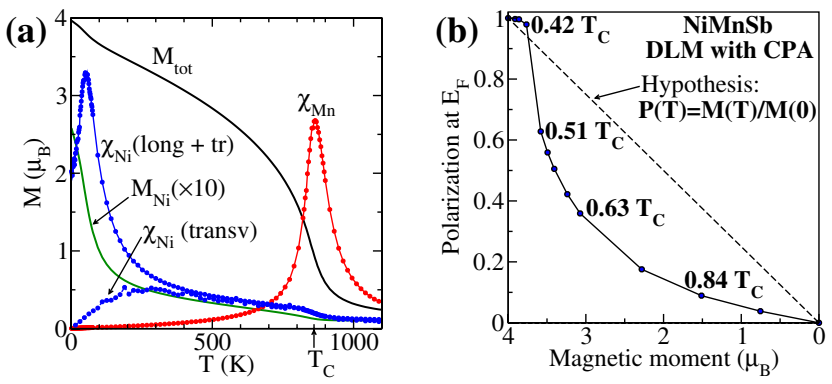

FIG. 2 (color online). (a) Monte Carlo results for the $T$-dependent magnetic properties. $M_{\mathrm{Ni}}$ (magnified by a factor 10) drops fast at $50 \mathrm{~K}$, fluctuating transversely and longitudinally; a susceptibility peak $\left[\chi_{\mathrm{Ni}}(\right.$ long + tr $\left.)\right]$ is evident. If only transverse fluctuations are allowed, $\chi_{\mathrm{Ni}}$ (transv) has a broad maximum. (b) $P$ at $E_{F}$ as function of total spin moment for the DLM states for $\mathrm{NiMn}_{1-x}^{\uparrow} \mathrm{Mn}_{x}^{\downarrow} \mathrm{Sb}$ calculated with the CPA. The degree $x$ of disorder increases from left $(x=0)$ to right $(x=$ $0.5)$, simulating the increase of $T$ from 0 to $T_{C}$. tudinal fluctuations (essential to our model) on the $\mathrm{Ni}$ sublattice allow for a high rate of energy absorption around the crossover $(50 \mathrm{~K})$, the classical treatment of the $\mathrm{Ni}$ sublattice is applicable already at such low $T$. We believe the peak of $\chi_{\mathrm{Ni}}$ and the drop of $M_{\mathrm{Ni}}$ at low $T$ to be connected to the so-far unexplained experimental findings of an anomaly in the temperature dependence of the magnetization and the resistivity at approximately $80 \mathrm{~K}$ [23]. Assuming that the magnetic moments on the Ni sublattice are disordered, the system can dissipate energy at a higher rate leading to an increase in resistance.

Despite the loss of short-range order in the Ni sublattice, mechanism (2) of local noncollinearity does not affect the polarization significantly. Since the gap originates from the $d-d$ hybridization between $\mathrm{Ni}$ and $\mathrm{Mn}$ spin-down states, and since the $\mathrm{Ni} d$ states are below $E_{F}$ for both spin directions, a rotation of the $\mathrm{Ni}$ moment causes a hybridization of the $\mathrm{Mn} d$ spin-down states partly with the $\mathrm{Ni}$ spin-up states and partly with the Ni spin-down states. Consequently, the $d$ - $d$ hybridization remains, but the gap is reduced. The relevant DOS is shown in Fig. 1(b), for the case of the Mn moments remaining in a ferromagnetic configuration, while the $\mathrm{Ni}$ moments are constrained to a $60^{\circ}$ angle relative to $\mathrm{Mn}$ (whence their magnitude is reduced to $0.16 \mu_{B}$ ). The reduction of the gap follows from a reduction of the hybridization strength: as the Ni moment is reduced, its spin-down states move lower in energy and hybridize less with the Mn spin-down states. Thus, the spin-down "conduction band" ultimately reaches $E_{F}$ and half-metallicity is lost. This is the signature of the hybridization mechanism (3), and comes on top of the noncollinear behavior of the Mn atoms [mechanisms (1) and (2)], which is weak at such low $T$ since the average Mn moment is still high, dictated by Bloch's $T^{3 / 2}$ law. At higher temperatures the locally noncollinear mechanism (2) should appear for Mn. The inset of Fig. 1(b) shows the state when $\mathrm{Mn}$ moments of neighboring layers form an angle of $50^{\circ}$ with respect to each other. Although mechanism (2) produces a mild effect of a finite spin-down DOS at $E_{F}$, a protrusion appears just $0.2 \mathrm{eV}$ under $E_{F}$. This is again related to mechanism (3), which is now triggered by the noncollinear configuration, and can cause a collapse of $P$ if the protrusion reaches $E_{F}$.

A quantitative estimate of the polarization as function of $T$ or of the order parameter $M$ requires knowledge of the response of the electronic structure to the increase of $T$. We achieve this by proceeding via mean-field theory, with the disordered local moment (DLM) state [11] representing the system at $T>0$ in a mean-field manner. A Mn site can then have a "down" orientation $\mathrm{Mn}_{x}^{\downarrow}$ (opposite to the magnetization) with probability $x$, or an "up" orientation $\mathrm{Mn}_{1-x}^{\dagger}$ (parallel to the magnetization). The ferromagnetic ground state $(T=0)$ corresponds to $x=0$, while the Curie point corresponds to $x=0.5$. The electronic structure of the DLM state for each $x$ is found within the coherent potential approximation (CPA), utilizing the Korringa-Kohn- 
Rostoker full-potential Green function method within DFT. This yields the average magnetic moment $M(x)$ [approximately, $M(x)=M(0)(1-2 x)$ so $x$ is an alternative order parameter] and the polarization $P(x)$.

We study the DLM states, $\mathrm{NiMn}_{1-x}^{\uparrow} \mathrm{Mn}_{x}^{\downarrow} \mathrm{Sb}$, with varying concentration $0<x<0.5$. One can directly check the hypothesis $P(T) \sim M(T)$ [9]. As shown in Fig. 2(b), such a relation does not hold. Instead, from a certain $M$ on, $P$ drops much faster than $M$. The reason can be traced back to the change in hybridization as $x$ (or $T$ ) increases: each $\mathrm{Ni}$ atom has on average $4(1-x) \mathrm{Mn}^{\dagger}$ neighbors and $4 x \mathrm{Mn}^{\downarrow}$ neighbors. The occupied $d$ states of the latter hybridize with the occupied spin-down states of Ni, push them higher in energy, and diminish the gap; $E_{F}$ reaches finally the valence band and $P$ collapses. An approximation of $M(T)$ within mean-field theory can be found by the use of the Brillouin function $B_{j}$. Doing this for $j=5 / 2$, we assigned the temperature values shown in Fig. 2(b). We see that, up to a "collapse temperature" $T_{0}=0.42 T_{C}$ (about room temperature), $P\left(E_{F}\right)$ remains close to $100 \%$, but then it drops fast, e.g., $P \approx 35 \%$ at $T=0.67 T_{C}$ (for $j=2, T_{0}=$ $0.45 T_{C}$ ). The globally noncollinear mechanism (1), not captured by the CPA, should be present on top of this behavior. Therefore, the initial plateau of $P$ (up to $T_{0}$ ) should be corrected towards a linear drop as $P(T)=$ $M(T) / M(0)$.

In summary, we have investigated the behavior of halfmetallic ferromagnets at elevated temperatures, with an emphasis on the properties of the gap and the spin polarization at $E_{F}$. We introduced an extended Heisenberg model including both transversal and longitudinal magnetic fluctuations, to treat multicomponent magnets which include subsystems of large moments coexisting with paramagnetic subsystems exhibiting a Stoner-enhanced susceptibility. The parameters entering the model were determined from first principles. We also estimated the polarization at $E_{F}$ within a CPA averaging. As prototypical system we have chosen the half-Heusler compound $\mathrm{NiMnSb}$. Our conclusions are the following: (i) In $\mathrm{NiMnSb}$, the Ni sublattice is weaker coupled than the $\mathrm{Mn}$ one; longitudinal fluctuations of the $\mathrm{Ni}$ moment are energetically as important as transversal fluctuations. This leads to an early crossover behavior of the magnetization at $T \approx 0.06 T_{C}$ where the average Ni moment is lost. (ii) The hybridization creating the gap is still present but the fluctuations change its strength. At this stage the gap width is reduced. (iii) At higher $T$, fluctuations of the Mn moments introduce a low DOS into the gap leading to a mild reduction of $P$, and, after a point, the hybridization changes so much that $E_{F}$ crosses the band edges. This is when the polarization collapses (around $0.42 T_{C}$ within mean-field theory). The behavior of $P$ shown here seems rather general for half- and full-Heusler alloys exhibiting halfmetallic ferromagnetism as we found by additional calculations. For example, for $\mathrm{Co}_{2} \mathrm{MnSi}, P$ was close to $100 \%$ till $0.27 T_{C}$, then it dropped fast, changed sign at $0.63 T_{C}$ and went back to zero at $T_{C}$. The decisive factor for the thermal collapse of polarization is the change in hybridizations due to the moment fluctuations; the effect of noncollinearity [9] is much milder. This work calls for experimental efforts to measure the sublattice magnetization and the spin polarization at $E_{F}$ as function of $T$ for half-metals.

We are grateful to P. H. Dederichs for enlightening discussions, and to H. Ebert and V. Popescu for providing us with their CPA algorithm [24].

*Electronic address: M.Lezaic@fz-juelich.de †Electronic address: Ph.Mavropoulos@fz-juelich.de

[1] R.A. de Groot, F. M. Mueller, P.G. van Engen, and K. H. J. Buschow, Phys. Rev. Lett. 50, 2024 (1983).

[2] I. Galanakis, P. H. Dederichs, and N. Papanikolaou, Phys. Rev. B 66, 134428 (2002); 66, 174429 (2002).

[3] Ph. Mavropoulos et al., Phys. Rev. B 69, 054424 (2004).

[4] S. J. Jenkins and D. A. King, Surf. Sci. 494, L793 (2001); I. Galanakis et al., Phys. Rev. B 71, 214431 (2005).

[5] T. Block et al., Phys. Rev. B 70, 205114 (2004).

[6] D. Orgassa et al., Phys. Rev. B 60, 13237 (1999); S. Picozzi et al., Phys. Rev. B 69, 094423 (2004).

[7] These arise from quantum fluctuations due to the on-site Coulomb interaction. Their effect on $P(T)$ was studied by L. Chioncel et al., Phys. Rev. Lett. 96, 137203 (2006).

[8] K. E. H. M. Hanssen et al., Phys. Rev. B 42, 1533 (1990); R. J. Soulen, Jr. et al., Science 282, 85 (1998); M. Bowen et al., Phys. Rev. Lett. 95, 137203 (2005).

[9] In a crude approximation, $P(T) \approx M(T) / M(0)$, as suggested (in an equivalent form) by R. Skomski and P. A. Dowben, Europhys. Lett. 58, 544 (2002).

[10] S. V. Halilov et al., Phys. Rev. B 58, 293 (1998).

[11] B. L. Gyorffy et al., J. Phys. F 15, 1337 (1985).

[12] E. Şașioğlu et al., Phys. Rev. B 72, 184415 (2005).

[13] J. Kübler, Phys. Rev. B 67, 220403 (2003); Y. Kurtulus et al., Phys. Rev. B 71, 014425 (2005).

[14] J. Rusz et al., Phys. Rev. B 73, 214412 (2006).

[15] Ph. Kurz et al., Phys. Rev. B 69, 024415 (2004).

[16] Based on the time scale of magnons being much slower than electron hopping, whence the electron gas has time to relax under the constraint of the presence of a magnon.

[17] http://www.flapw.de

[18] J. P. Perdew et al., Phys. Rev. Lett. 77, 3865 (1996).

[19] D.P. Landau and K. Binder, A Guide to Monte Carlo Simulations in Statistical Physics (Cambridge University Press, Cambridge, England, 2000).

[20] The approach by Mryasov et al. [Europhys. Lett. 69, 805 (2005)] on FePt alloys does not take $M_{\mathrm{Pt}}$ to be a separate degree of freedom. In NiMnSb, the high susceptibility of $\mathrm{Ni}$ does not allow for a similar approximation.

[21] Ch. Hordequin, E. Lelievre-Berna, and J. Pierre, Physica (Amsterdam) 234B-236B, 602 (1997).

[22] C. N. Borca et al., Phys. Rev. B 64, 052409 (2001).

[23] C. Hordequin, J. Pierre, and R. Currat, J. Magn. Magn. Mater. 162, 75 (1996).

[24] http://olymp.cup.uni-muenchen.de/ak/ebert/SPR-TBKKR 